\title{
Arthrotomy and Lavage of Neonatal Septic Arthritis - A Multicenteric Study
}

\section{Madhan J ${ }^{1 *}$, Naveen J², Eswar $\mathbf{R}^{3}$ and Raam Mohan $\mathrm{S}^{4}$}

${ }^{1}$ Senior Resident, Department of Orthopaedics, School of Medical Sciences and Research, India

2Junior Resident, Department of Orthopaedics, Kasturba Medical College, India 3Junior Resident, Department of Orthopaedics, Madras Medical College, India

\section{Research Article}

Volume 3 Issue 3

Received Date: June 12, 2019

Published Date: July 16, 2019

DOI: $10.23880 /$ jobd- 16000186 ${ }^{4}$ Junior Resident, Department of Orthopaedics, Mohana Kumaramangalam Medical College, India

*Corresponding author: Dr. Madhan Jeyaraman, Senior Resident, Department of Orthopaedics, School of Medical Sciences and Research, Sharda University, Uttar Pradesh, India. Tel: +91 8310600785; Email: madhanjeyaraman@gmail.com

\section{Abstract}

Background: Acute septic arthritis (SA) of childhood is a potentially devastating disease that may cause permanent disability or even death, especially in resource-limited settings. Septic arthritis is rare in developed countries, the annual incidence being 4 cases per 1,00,000 but is considerably more common in lower socioeconomic groups.

Objective: To study about the septic arthritis in neonates using arthrotomy \& lavage and the complications associated with septic arthritis.

Materials and Methods: A total of 319 neonates with septic arthritis irrespective of the joint involved, admitted to NICU in School of Medical Sciences and Research, Madras Medical College and Mohan Kumaramangalam Medical College for a period of three consecutive years were taken up for the study. All suspected cases of neonatal septic arthritis were subjected for detailed clinical history, examination, hematological and radiological investigations were done. All suspected cases were started with empirical or culture specific IV antibiotics for 2 weeks. The neonates who responded to the treatment given within $48-72$ hours were treated conservatively and excluded from our study. The neonates who did not respond to the antibiotics given were subjected for arthrotomy and lavage of the affected joint. The aspirated material was sent for culture and sensitivity for specific antibiotic treatment. The treatment was given with culture specific IV antibiotics for a minimum period of 2 weeks followed by oral antibiotics for a period of 2 weeks from the time symptoms subsides. All cases were followed up at a monthly interval to a minimum period of 1 year of their age.

Results: A total of 512 septic arthritis cases were seen in infants of whom 319 cases were neonates during the three year study period. The most common organism causing neonatal septic arthritis in our study was Staphylococcus aureus in 116 cases (36.36\%). No organism was cultured in 81 cases $(25.39 \%)$. Viral or fungal or anaerobes or reactive arthritis might be responsible for this $25.39 \%$ cases. Concomitant osteomyelitis was found in 5 cases in hip $\% 1$ case 


\section{Journal of Orthopedics \& Bone Disorders}

in knee. Tomsmith arthritis of hip was found in 2 cases. Mild joint stiffness were found in 7 cases in hip, 3 cases in

knee, 1 case in shoulder and 1 case in ankle seen at follow up. After discharge gentle passive joint motion were advised. The missed cases from follow up were not included in the study.

Conclusion: The sequelae of septic arthritis in neonates can be prevented by early diagnosis and early intervention with emergency lavage of any involved joint to reduce the rate of disability. The arthrotomy and lavage is the definitive treatment for neonatal septic arthritis which reduce the morbidity and mortality of the neonates.

Keywords: Septic Arthritis; Arthrotomy; Lavage; Tomsmith; Staphylococcus aureus

\section{Introduction}

Septic arthritis (SA) is a frequent concomitant of osteomyelitis of the long bones. Occasionally septic arthritis occurs in the absence of demonstrable osteomyelitis, in which case the pathogenesis is either direct haematogenous seedling of the joint (primary septic arthritis) or secondary to occult osteomyelitis. Staphylococcus aureus is the commonest single cause of all cases of primary septic arthritis (45\%) but gram negative bacilli $(25 \%)$ causes proportionately more cases than of osteomyelitis [1].

Acute septic arthritis (SA) of childhood is a potentially devastating disease that may cause permanent disability or even death, especially in resource-limited settings. Septic arthritis is rare in developed countries, the annual incidence being 4 cases per 1,00,000, but is considerably more common in lower socioeconomic groups $[2,3]$. There is a small male preponderance, with a male-tofemale ratio of around 1.73 and a slight increase in frequency at school age [4]. Although there is general agreement that it is urgent to treat septic arthritis in children, the method for draining and lavage differs from one team to another. Puncture drainage, needle aspiration-lavage and draining by arthroscopy or arthrotomy are the most frequently described techniques $[5,6]$.

\section{Objective}

To study about the septic arthritis in neonates using arthrotomy \& lavage and the complications associated with septic arthritis.

\section{Materials and Methods}

A multicenteric prospective clinical study with a total of 319 neonates with septic arthritis irrespective of the joint involved, admitted to NICU in School of Medical Sciences and Research, Madras Medical College and Mohan Kumaramangalam Medical College for arthrotomy and lavage, for a period of three consecutive years were taken up for the study. In all three centers, the same treatment protocol was followed up.

All the neonates were subjected for detailed clinical examination like swelling, redness, tenderness along with limitation of movements in the joint affected. The radiological analysis for all suspected cases of septic arthritis in neonates using detailed clinical history and examination, on admission, routine basic investigations including complete blood count, ESR at 1 hour, CRP, blood culture and sensitivity were done. Based on the primary cause of admission to NICU, culture \& sensitivity of urine, stool and CSF were sent to rule out secondary causes of septic arthritis.

The radiograph of the affected joint to look for widening of the joint space \& bone destruction and ultrasonogram of the affected joint to look for any joint effusion were done subsequently. The ultrasonogram for joint effusion, clinical examination finding suggesting septic arthritis like swelling, redness, tenderness along with limitation of movements in the joint affected was taken as the main diagnostic criteria in our study.

All suspected cases were started with empirical or culture specific IV antibiotics. The neonates who responded to the treatment given within 48-72 hours were treated conservatively and excluded from our study. The neonates who did not respond to the antibiotics given were subjected for arthrotomy and lavage of the affected joint. The aspirated material was sent for culture and sensitivity for specific antibiotic treatment.

The IV antibiotic therapy was initiated with a combination of ampicillin, cefotaxime and cloxacillin. This 


\section{Journal of Orthopedics \& Bone Disorders}

regimen was altered according to the culture and sensitivity results of joint fluid. The treatment was given with specific IV antibiotics for a minimum period of 2 weeks followed by oral antibiotics for a period of 2 weeks from the time symptoms subsides. As the clinical symptoms like tenderness, warmth, swelling around the joint subsides; mother or the attenders were taught for gentle passive movement of the joint to prevent stiffness and atrophy around the joint. All cases were followed up at a monthly interval to a minimum period of 1 year of their age.

\begin{tabular}{|c|c|c|}
\hline \multicolumn{3}{|c|}{$\begin{array}{c}\text { Suspected neonatal septic arthritis } \\
\downarrow \\
\downarrow \\
\text { Detailed history \& clinical examination } \\
\downarrow \\
\text { \& ESR estimation and USG of the affected joint } \\
\end{array}$} \\
\hline \multicolumn{2}{|c|}{$\begin{array}{c}\mathrm{CRP}<20 \mathrm{mg} / \mathrm{L} \\
\text { ESR }<20 \mathrm{~mm} / \mathrm{hr} \\
\text { No evidence of effusion on USG } \\
\downarrow \\
\text { Empirical IV antibiotics } \\
\downarrow \\
\text { Clinical observation }\end{array}$} & $\begin{array}{c}\text { CRP }<20 \mathrm{mg} / \mathrm{L} \\
\text { ESR }<20 \mathrm{~mm} / \mathrm{hr} \\
\text { No evidence of effusion on USG } \\
\downarrow \\
\text { Arthrotomy } \\
\downarrow \\
\text { Aspiration of pus or synovial fluid from the }\end{array}$ \\
\hline $\begin{array}{c}\text { Recovery } \\
\quad \downarrow \\
\text { Discharge }\end{array}$ & $\begin{array}{c}\text { Persistent symptoms } \\
\downarrow \\
\text { CRP \& ESR estimation }\end{array}$ & Joint fluid analysis and culture \\
\hline
\end{tabular}

Figure 1: Management plan for a child with suspected septic arthritis.

\section{Results}

A total of 319 cases of neonatal septic arthritis cases were taken into consideration for statistical analysis. The descriptive analytical statistics were evaluated statistically with IBM SPSS Statistics for Windows, Version 20.0, IBM Corp, Chicago, IL.

Out of 512 cases of septic arthritis, 193 cases (60.50\%) were male babies and 126 cases (39.49\%) were female babies with male to female ration of 1.5:1. Of the 319 cases, 203 cases $(63.63 \%)$ were delivered at our same institute; remaining 116 cases $(36.36 \%)$ were referred to our institutes. A total of 223 cases $(69.90 \%)$ were delivered by birth naturale and 96 cases (30.09\%) by LSCS for various obstetrical reasons. A total of 39 cases (45.34\%) had low birth weight of less than 2500 grams of which, majority of 21 cases $(24.41 \%)$ were of $2000-2500$ grams weight range.

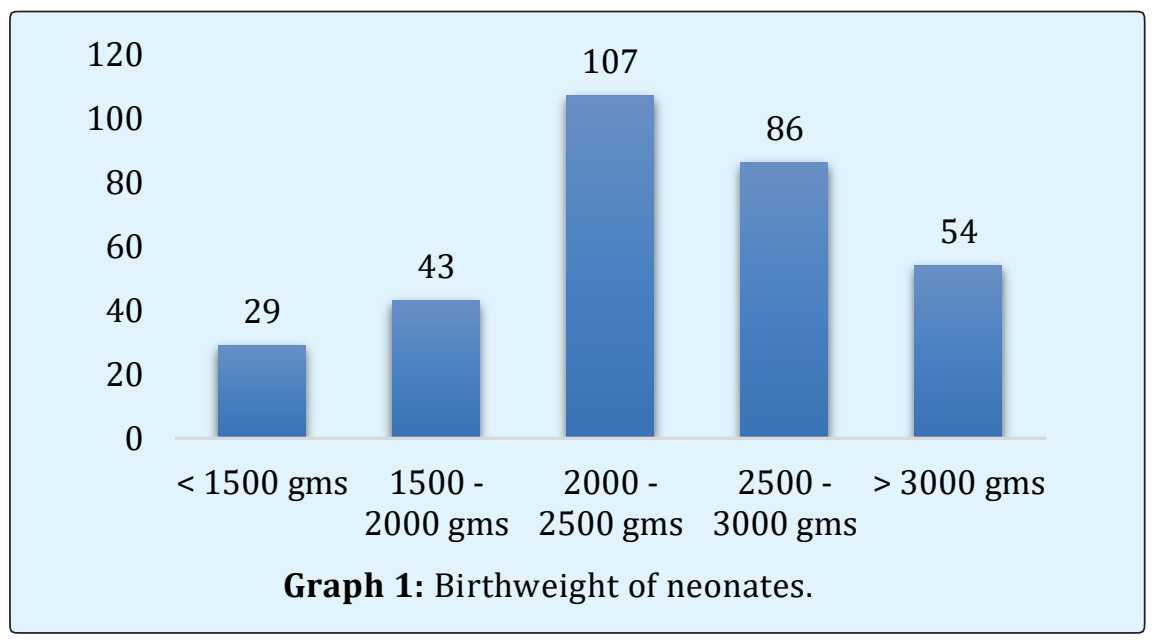

Madhan J, et al. Arthrotomy and Lavage of Neonatal Septic Arthritis - A Multicenteric Study. J Ortho Bone Disord 2019, 3(3): 000186. 


\section{Journal of Orthopedics \& Bone Disorders}

Excessive cry, refusal to feeds, rise in body temperature (axillary), decreased movement of the affected limb (pseudo paralysis) were the chief complaints noted in most of the neonates. Fever $>36.8^{\circ} \mathrm{C}$ (98.4 F), warmth, swelling around the joint and increased cry on moving the affected limb were the signs observed in most neonates which made to suspect septic arthritis and further investigations to confirm the diagnosis. All neonates were NICU admitted cases for various reasons. Out of 319 cases, a total of 279 cases $(87.46 \%)$ have got NICU admission prior to the development of arthritis which is shown in graph 2 . No cause was found in 40 cases $(12.53 \%)$

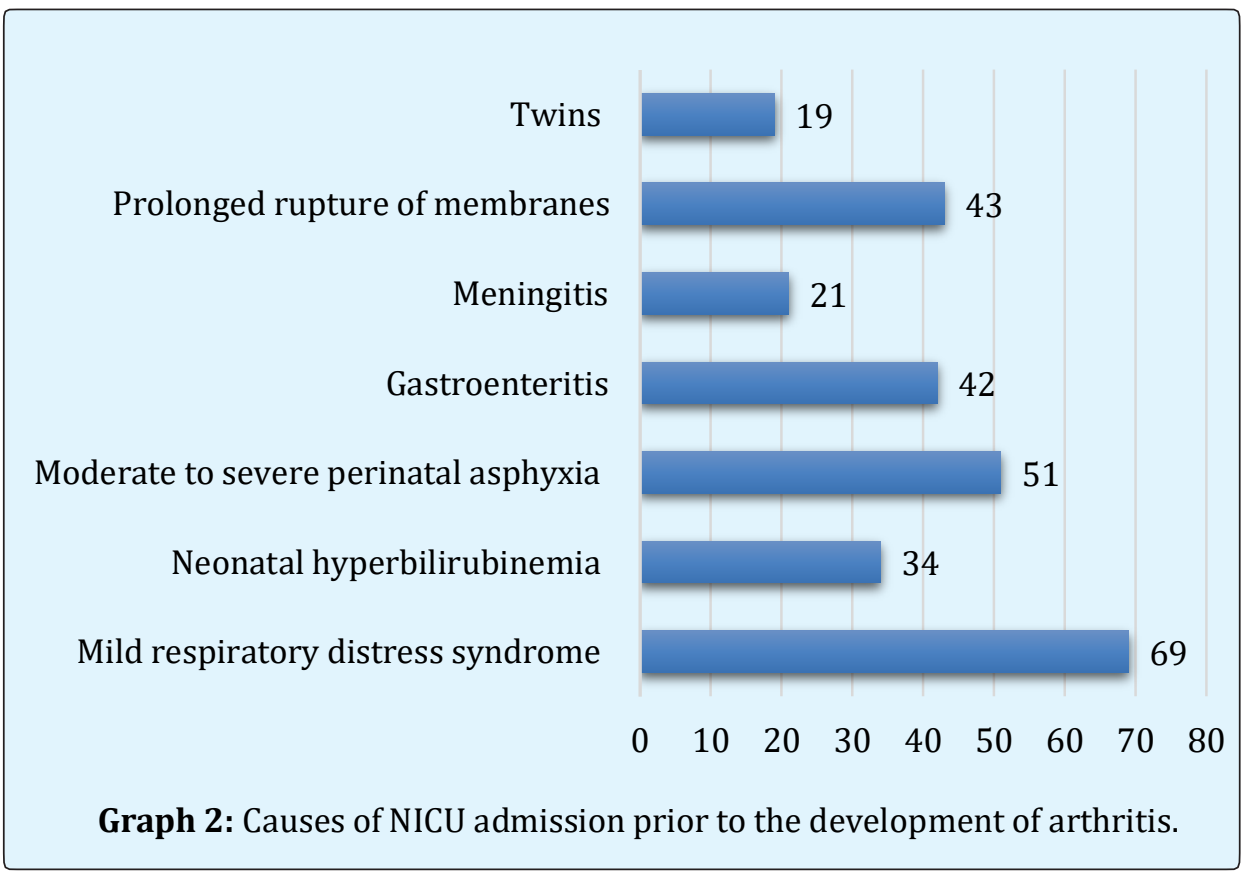

The most common joint affected in our study was hip in 177 cases $(55.48 \%)$ followed by knee in 119 cases

(37.30\%), shoulder in 19 cases (5.95\%) and ankle in 4 cases $(1.25 \%)$.

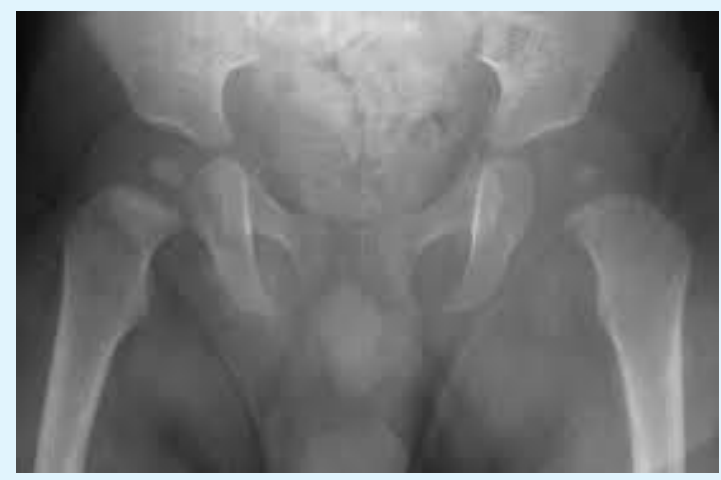

Figure 2: Radiograph of pelvis with B/L hips in AP view showing widening of joint space of left hip. 


\section{Journal of Orthopedics \& Bone Disorders}

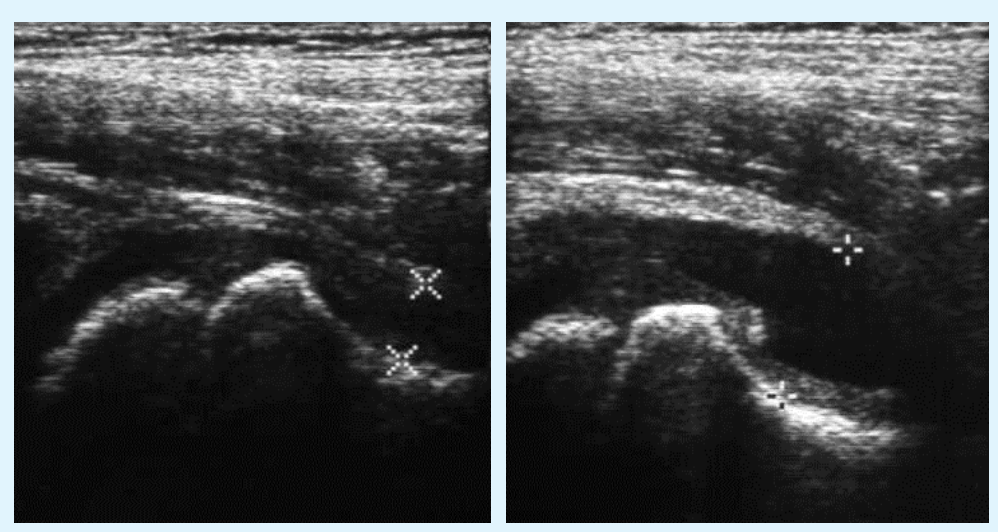

Figure 3: Ultrasonogram of right hip showing widening joint space of right hip.

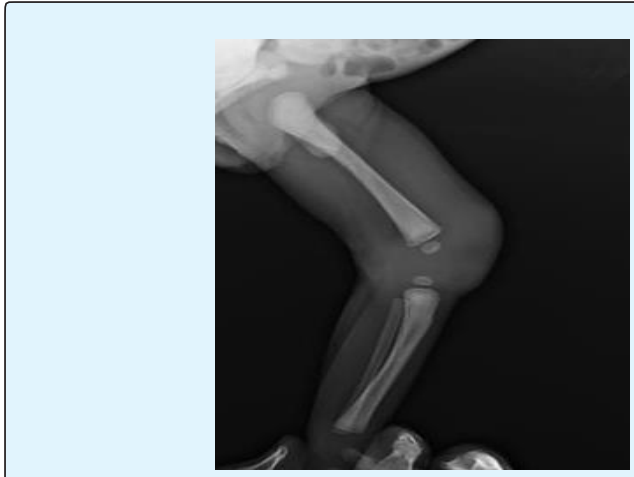

Figure 4: Radiograph of left knee lateral view showing soft tissue swelling around left knee with changes noted over left distal femur.

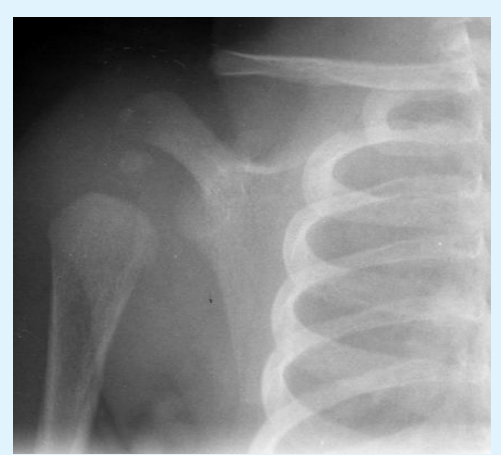

Figure 5: Radiograph of right shoulder AP view showing increased joint space over right shoulder joint.

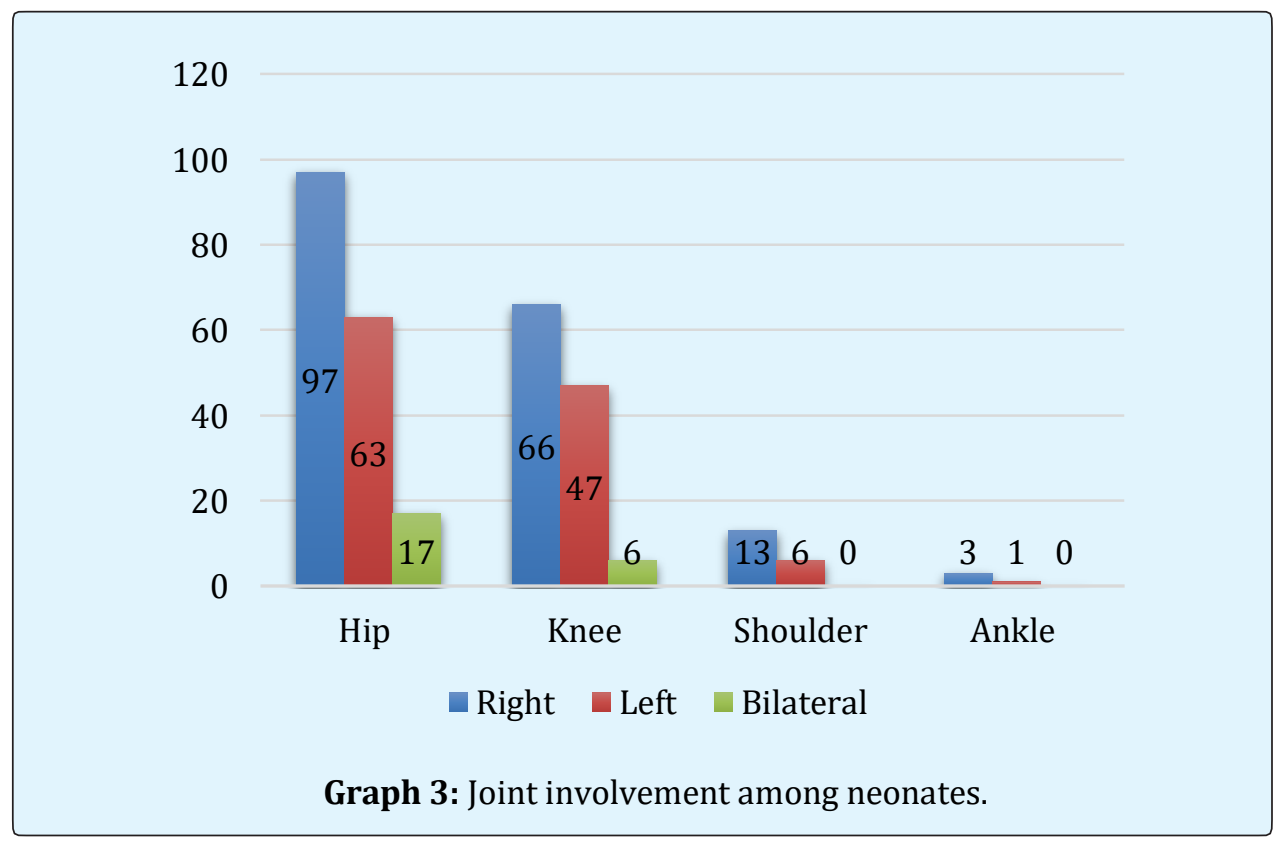

Madhan J, et al. Arthrotomy and Lavage of Neonatal Septic Arthritis - A Multicenteric Study. J Ortho Bone Disord 2019, 3(3): 000186. 


\section{Journal of Orthopedics \& Bone Disorders}

All suspected neonatal septic arthritis cases have been screened hematologically with a panel of tests like ESR, CRP and PMN. $100 \%$ of cases reported ESR $>20 \mathrm{~mm} / \mathrm{hr}$, $86.21 \%$ of cases reported CRP $>20 \mathrm{mg} / \mathrm{L}$ and $75.83 \%$ of cases reported PMN $>80 \%$. Ultrasonogram (USG) was the main diagnostic investigation in our study. The fluid collection in joints (hip, knee, shoulder and ankle) was seen in all 319 cases (100\% sensitivity and 92\% specificity).

Blood culture showed positive growth for Staphylococcus aureus in 198 cases (62.06\%), Escherichia coli in 36 cases (11.28\%) and no growth in 85 cases (26.64\%). For 4 cases which showed negative joint fluid culture and positive blood culture was due to starting of IV antibiotics according to blood culture sensitivity report. The negative cultures from joint and blood is mostly because of the starting of IV antibiotics in NICU for primary cause or the empirical antibiotics which was started conservatively for $48-72$ hours to wait for the subsidence of symptoms and to consider for arthrotomy \& lavage if not subsided.

All 319 cases underwent arthotomy and lavage under general anaesthesia. The pus was found in 127 joints (39.81\%) totally. Among 319 cases, WBC count > $50,000 / \mathrm{mm}^{3}$ was found in 181 cases $(56.73 \%)$. However, distinguishing septic arthritis from various aseptic arthritides through synovial fluid analysis has always been problematic [7]. The most common organism causing neonatal septic arthritis in our study was Staphylococcus aureus in 116 cases (36.36\%). No organism was cultured in 81 cases (25.39\%). Viral or fungal or anaerobes or reactive arthritis might be responsible for this $25.39 \%$ cases.

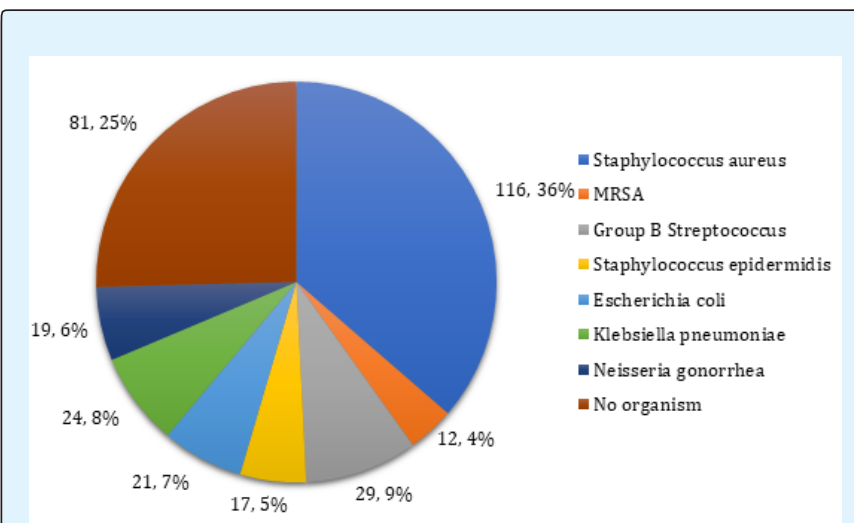

Graph 4: Causative organism from the joint fluid culture analysis.

After discharge gentle passive joint motion was advised. All cases were followed up at a monthly interval to a minimum period of 1 year of their age Missed cases from follow up were not included in the study.

\begin{tabular}{|c|c|c|c|c|c|}
\hline \multirow{2}{*}{ Clinical parameters } & \multicolumn{4}{|c|}{ Mean \pm SD } & \multirow{2}{*}{$P$ value } \\
\hline & During admission & $1^{\text {st }}$ month & $6^{\text {th }}$ month & $12^{\text {th }}$ month & \\
\hline $\begin{array}{l}\text { WBC count } \\
\text { (cells } / \mathrm{mm}^{3} \text { ) }\end{array}$ & $13481.35 \pm 1265.25$ & $8600.42 \pm 932.67$ & $7213.12 \pm 759.32$ & $5712.38 \pm 452.75$ & $<0.001$ \\
\hline $\begin{array}{l}\text { Neutrophil count } \\
\text { (cells } / \mathrm{mm}^{3} \text { ) }\end{array}$ & $8914.63 \pm 1847.78$ & $6724.51 \pm 784.62$ & $5713.52 \pm 562.90$ & $4418.93 \pm 320.34$ & $<0.001$ \\
\hline CRP (mg/L) & $62.14 \pm 11.62$ & $16.63 \pm 7.83$ & $6.01 \pm 1.02$ & $4.21 \pm 0.78$ & $<0.001$ \\
\hline ESR @ 1hr (mm/hr) & $39.16 \pm 11.74$ & $22.38 \pm 7.26$ & $19.15 \pm 6.18$ & $11.34 \pm 3.17$ & $<0.001$ \\
\hline Body temp.(F) & $101.71 \pm 1.97$ & $99.64 \pm 1.26$ & $98.23 \pm 0.45$ & $98.10 \pm 0.01$ & $<0.001$ \\
\hline
\end{tabular}

Table 1: Progression of clinical parameters.

The Student t-test and Chi-square tests were used for statistical analysis. We found a significant statistical difference in clinical parameters between the cases during admission and follow up during $12^{\text {th }}$ month post op $(\mathrm{p}<0.001)$.

Concomitant osteomyelitis was found in 5 cases $(2.82 \%)$ in hip and 1 case $(0.84 \%)$ in knee who resolved with arthrotomy lavage followed by IV antibiotics given according to culture sensitivity of the joint fluid analysis. 2 cases $(1.12 \%)$ had Tomsmith arthritis of hip at follow up. Mild joint stiffness were found in 7 cases $(3.95 \%)$ in hip, 3 cases $(2.52 \%)$ in knee, 1 case $(5.26 \%)$ in shoulder and 1 case $(25.0 \%)$ in ankle seen at follow up for which gentle passive physiotherapy was advised to increase the range of motion of the joint.

There was no limitation of movements of joints in all cases except for mild stiffness found in 7 cases $(3.95 \%)$ in hip, 3 cases $(2.52 \%)$ in knee, 1 case (5.26\%) in shoulder and 1 case $(25.0 \%)$ in ankle. We found a statistically significant difference in range of movements of involved 


\section{Journal of Orthopedics \& Bone Disorders}

joints between pre-operative period and 12 months post op $(\mathrm{p}<0.05)$.

\section{Discussion}

Modality of treatment: Though there are different modalities of treatment in treating septic arthritis like conservative, needle aspiration, arthroscopic lavage, arthrotomy lavage, we have chosen the arthrotomy and lavage which most of the authors believe as the definitive treatment for septic arthritis. Though needle aspiration is the simplest and least invasive, especially in deep joint like hip, it has the disadvantage of inability to lavage the debris completely. So, most authors prefer arthrotomy for debridement of all septic arthritic joint [7-9]. The choice of technique has long been controversial. In 1979, Lunseth and Heiple [10] did not find any difference in results between arthritis treated by arthrotomy or simple aspiration. There is general agreement that treatment must include draining the joint sufficiently to mechanically eliminate a maximum of fungal debris followed by antibiotic treatment. Difficulty in draining the synovial fluid suggests the presence of pseudo membranes, which cannot be removed by needle aspiration, and can be done only by arthroscopy or arthrotomy. As this study is particularly on neonates and difficulty in using arthroscopic probes in small joint area and also it needs skilled surgical team, we preferred arthrotomy lavage for all the cases.

Criteria for diagnosis: There are no universally accepted criteria, but an acute illness in a neonate with a warm and painful joint and limited motion suggests septic arthritis. The rise in body temperature, excess cry, refusal to feeds, warmth and swelling around the joint, decreased movement of the affected joint are clinical criteria taken for suspected septic arthritis in our study. Erythema and edema of the skin and soft tissue overlying the site of infection are seen earlier in septic arthritis than in osteomyelitis, because the bulging infected synovium is usually more superficial, whereas the metaphysis is located more deeply. Septic arthritis in hip is an exception because of the deep location of the hip joint [11-14].

Biological Investigations: Increased CRP, ESR \& WBC cell count particularly polymorphs are the biological criteria showing the presence of infection. Ultrasonogram (USG) was the most useful tool of radiological investigation showing the joint effusion and fluid in soft tissue around the joint directing our focus towards infection in the affected joint. A child with possible septic arthritis presenting with CRP $<20 \mathrm{mg} / \mathrm{l}$ and ESR $<20$ $\mathrm{mm} / \mathrm{hr}$ is very unlikely to have the disease [12] but if one (or both) of these parameters is increased, arthrocentesis is indicated, mainly to obtain a representative sample for bacteriology. ${ }^{11}$ CRP and ESR were raised mostly in all patients. CRP is time sensitive and is a disease progress indicator, it rises immediately after infection and when antibiotics started and infection settles down, its values comes down. It shows downward trend as soon as third day after arthrotomy. But it takes 6 - 7 days for ESR to fall down which may stretch for a long period of time. So, CRP is more sensitive than ESR in monitoring the response of treatment. It was well supported by Tachdijan [13]. The biological investigations were repeated on day 12, before stopping IV antibiotics which shows the fall in CRP, ESR, leukocyte and neutrophil count drastically, though in many patients it was on downfall track.

Radiological investigations: Ultrasonogram is highly sensitive in detecting joint effusion particularly for the hip joint, where plain radiographs are normal in more than $50 \%$ cases of septic arthritis of the hip [14]. The sensitivity of sonography for detecting joint effusion is $90 \%-100 \%$ [15]. The echogenicity of the fluid is variable and fluid can appear anechoic, hypoechoic or hyperechoic $[15,16]$. Thus USG is taken as the most valuable investigation in our study to confirm the septic arthritis of the suspected joint. All cases having joint effusion were subjected to arthrotomy lavage. The remaining cases which didn't have effusion were given only conservative treatment with empirical antibiotics.

Blood culture showed positive growth for Staphylococcus aureus in 198 cases (62.06\%), Escherichia coli in 36 cases $(11.28 \%)$ and no growth in 85 cases (26.64\%). For 4 cases which showed negative joint fluid culture and positive blood culture was due to starting of IV antibiotics according to blood culture sensitivity report. The negative cultures from joint and blood is mostly because of the starting of IV antibiotics in NICU for primary cause or the empirical antibiotics which was started conservatively for 48-72 hours to wait for the subsidence of symptoms and to consider for arthrotomy \& lavage if not subsided.

Synovial fluid analysis for TC, DC, glucose has limited usefulness because non-infectious inflammatory diseases, such as RF, and RA, can also show exuberant reaction with increased cells and proteins and decreased glucose. Nevertheless cell counts $>50,000-1,00,000$ cells $/ \mathrm{mm}^{3}$ generally indicate an infectious process [14]. In our study, among the 54 arthrotomy joints, pus was found in 127 joints $(39.81 \%)$ totally. WBC count $>50,000$ cells $/ \mathrm{mm}^{3}$ was found in 181 cases (56.73\%). The positive joint 


\section{Journal of Orthopedics \& Bone Disorders}

fluid culture was found in 238 cases $(74.60 \%)$. Among these culture analyses, Staphylococcus aureus was seen in 116 cases (36.36\%) which emerged as the most common causative organism for septic arthritis in neonates. Staphylococcus aureus is now the most common cause of septic arthritis in all groups [13,14,17].

Joint involvement: Hip (177 cases, 55.48\%) is the most common joint affected in our study followed by knee (119 cases, $37.30 \%$ ), then by shoulder (19 cases, $5.95 \%$ ) and ankle ( 4 cases, $1.25 \%$ ). According to Gafur OA, et al. knee is the most common site in $40 \%$ and hip is the second most in $22-40 \%$ of neonates [18]. The source of bone infection is usually haematogenous but direct trauma during femoral and heel puncture, fetal monitoring electrodes, exchange transfusion or venous cut down have been reported as sources of infection [19]. Unilateral involvement of the hip joint occurred in $94 \%$ of our cases, in contrast to other reported series in which there was a high incidence of bilateral hip joint involvement [20].

Causes: 179 cases (56.11\%) out of 319 neonatal septic arthritis in our study were LBW < 2500 gms. Premature and LBW neonates need special care in NICU unit for survival; many procedures are needed to help them grow. These babies with underdeveloped immune system acquire bacteremia by various procedures like chronic indwelling catheters, IV access, and long hospital stay which subsequently lead to septic arthritis. Prematurity and respiratory distress syndrome have been reported as possible predisposing factors for septic arthritis [21]. 69 cases of our neonates suffered from mild respiratory distress syndrome and 51 cases suffered from perinatal asphyxia which might predispose to septic arthritis in our series.

Complications: According to the Welcon, et al. [22], the initiation of appropriate medical / surgical treatment four or more days after the onset of symptoms may be associated with poor outcomes. In the opinion of Rutz and Spoerri [23], a septic joint condition should always be treated as an emergency at all times. Totally 19 complicated septic arthritis were seen in our study. Concomitant osteomyelitis was found in 5 cases $(2.82 \%)$ in hip and 1 case $(0.84 \%)$ in knee who resolved with arthrotomy lavage followed by IV antibiotics given according to culture sensitivity of the joint fluid analysis. 2 cases $(1.12 \%)$ had Tomsmith arthritis of hip at follow up. Mild joint stiffness were found in 7 cases $(3.95 \%)$ in hip, 3 cases $(2.52 \%)$ in knee, 1 case $(5.26 \%)$ in shoulder and 1 case $(25.0 \%)$ in ankle seen at follow up for which gentle passive physiotherapy was advised to increase the range of motion of the joint. All these 19 cases were presented late for orthopaedic opinion for atleast 5 days since the onset of symptoms. The complications were seen only in such cases in which arthrotomy was planned with the delay of 5 days and not seen in other cases as arthrotomy was planned in less than 5 days. Whether osteomyelitis predisposed to septic arthritis or vice versa is not clear as both presented together in 2 cases. Primary septic arthritis is on the rise in contrast to secondary to osteomyelitis. We had only 5 cases with proximal femur metaphysis osteomyelitis, although the literature says septic arthritis is often associated with osteomyelitis $[13,24]$.

Follow up: After septic arthritis, follow-up for at least five years is necessary in order to detect complications, such as growth arrest or avascular necrosis $[25,26]$. This is the drawback of our study as the follow up in our series was till one year of age only in all joint cases. MRI scan of the affected joint is needed to study about the joint complication which we couldn't do in the follow up because of cost factor and non-affordability by the attenders. As most of the infants were not started walking at the follow up and also free of complaints, parents didn't agree for further investigations.

When it comes to the prognostic factors, a significant controversy can be seen in the literature. Is it age or delay in treatment which dictates poor outcomes? Research shows that the worst prognosis is seen in neonates; but we cannot confirm it as an independent factor from delay in diagnosis and treatment [26]. Also with this limited follow up period and without MRI follow up it is very difficult to comment on the real complication rate of the arthrotomy lavage cases in neonatal septic arthritis.

\section{Conclusion}

The sequelae of septic arthritis in neonates can be prevented by early diagnosis and early intervention with emergency arthrotomy lavage of any involved joint to reduce the rate of disability. Arthrotomy lavage is the definitive treatment for neonatal septic arthritis. Decision to open the joint should be taken as soon as the conservative treatment failed to relieve the symptoms and intra-articular pressure of the joint and to prevent the ischemic bony changes. The low complication rate in our series by arthrotomy shows arthrotomy lavage as the best treatment option, but its long term follow up need to be updated with MRI to validate the true complication rate. Counselling the patients regarding the importance of MRI follow up in patients with neonatal septic arthritis is mandatory for early diagnosis of sequelae and for 


\section{Journal of Orthopedics \& Bone Disorders}

initiation of further treatment accordingly. Following aseptic precautions in NICU is very must to decrease the incidence of septic arthritis in neonates admitted for special care for primary causes.

\section{References}

1. David Issac E, Richard Moxon (1991) Neonatal infections, $1^{\text {st }}$ (Edn.), chapter 9, pp: 94-95.

2. Peltola H, Paakkonen M, Kallio P, Kallio MJ (2009) Osteomyelitis-Septic Arthritis (OM-SA) Study Group. Prospective, randomized trial of 10 days versus 30 days of antimicrobial treatment, including short term course of parenteral therapy, for childhood septic arthritis. Clin Infect Dis 48(9): 1201-1210.

3. Lavy CB, Thyoka M, Pitani AD (2005) Clinical features and microbiology in 204 cases of septic arthritis in Malawian children. J Bone Joint Surg Br 87(11): 15451548.

4. Peltola H, Kallio MJT, Unkila-Kallio L (1998) Reduced incidence of septic arthritis in children by Haemophilus influenza type-b vaccination. Implications for treatment. J Bone Joint Surg $\mathrm{Br} 80$ : 471-473.

5. Smith SP, Thyoka M, Lavy CB, Pitani A (2002) The Blantyre Septic Joint Score: a new scoring system for septic arthritis. Trop Doct 32(4): 250-251.

6. El-Sayed AM (2008) Treatment of early septic arthritis of the hip in children: comparison of results of open arthrotomy versus arthroscopic drainage. J Child Orthop 2(3): 229-237.

7. Kortekangas P, Aro HT, Tuominen J (1992) Synovial fluid leucocytosis in bacterial arthritis vs reactive arthritis and rheumatoid arthritis in the adult knee. Scand J Rheumatol 21(6): 283-288.

8. Petersen S, Knudsen FU, Andersen EA, Egeblad M (1980) Acute haematogenous osteomyelitis and septic arthritis in childhood: a 10-year review and follow-up. Acta Orthop Scand 51(3): 451-457.

9. Nord KD, Dore D, Deeney VF, Armstrong AL, Cundy PJ, et al. (1995) Evaluation of treatment modalities for septic arthritis with histological grading and analysis of levels of uronic acid, neutral protease and interleukin 1. J Bone Joint Surg Am 77(2): 258-265.
10. Lunseth PA, Heiple KG (1979) Prognosis in septic arthritis of the hip in children. Clin Orthop 139: 8185.

11. Paakkonen M, Kallio MJ, Peltola H, Kallio PE (2010) Pediatric septic hip with or without arthrotomy: retrospective analysis of 62 consecutive nonneonatal culture-positive cases. J Pediatr Orthop B 19(3): 264269.

12. Paakkonen M, Kallio MJ, Kallio PE, Peltola H (2010) Sensitivity of erythrocyte sedimentation rate and Creactive protein in childhood bone and joint infections. Clin Orthop Relat Res 468(3): 861-866.

13. Herring JA (2001) Tachdijan's Paediatric Orthopaedics. $3^{\text {rd }}$ (Edn.), WB Saunders co, Philadelphia, pp: 1841-1877.

14. Kliegman Stanton, St Geme Schor (2017) Nelson Textbook of Pediatrics. First South Asian Edition, Chapter 685, Elsevier, New Delhi, pp: 3327-3330.

15. Zawin JK, Hoffer FA, Rand FF, Teele RL (1993) Joint effusion in children with an irritable hip: US diagnosis and aspiration. Radiology 187(2): 459-546.

16. Rohrschneider WK, Fuchs G, Troger J (1996) Ultrasonographic evaluation of anterior ascending recess in the normal hip: a prospective study of 166 asymptomatic children. Pediatric Radiol 26: 629-634

17. Chen CE, Ko JY, Li CC, Wang CJ (2001) Acute septic arthritis of the hip in children. Arch Orth Traum Surg 121(9): 521-526.

18. Gafur OA, Copley LA, Hollmig ST, Browne RH, Thornton LA, et al. (2008) The impact of current epidemiology of pediatric musculoskeletal infection on evaluation and treatment guidelines, J Pediatric Orthop 28(7): 777-785.

19. McGregor JA, McFarren T (1989) Osteomyelitis: a complication of fetal monitoring. Obstet Gynecol 73: 490-492.

20. Frederiksen B, Christiansen P, Knudsen FU (1993) Acute osteomyelitis and septic arthritis in the neonate, risk factors and outcome. Eur J Pediatr 152(7): 577-580.

21. Gillespie R (1973) Septic arthritis of childhood. Clin Orthop 96: 152-159 


\section{Journal of Orthopedics \& Bone Disorders}

22. Welcon CJ, Fisher MC, ALburger PD, Long SS (1986) Pyogenic arthritis in infants and childrens: a review of 95 cases. Pediatr Infecr Dis 5(6): 669-676.

23. Rutz E, Spoerri M (2013) Septic arthritis of the paediatric hip-A review of current diagnostic approaches and therapeutic concepts. Acta Orthop Belg 79(2): 123-134.

24. Paterson D (1978) Septic arthritis of the hip joint. Orthop clin north Am 9(1): 135-142.
25. Rutz E (2009) Brunner R. Septic arthritis of the hip current concepts. Hip Int 6: S9-S12.

26. Kariminasab MH, Shayesteh Azar M, Sajjadi Saravi M (2009) Surgical intervention for treatment of septic arthritis in infancy and childhood: a retrospective study. Arch Iran Med 12(4): 409-411. 